\title{
Observations of Flux Ropes With Strong Energy Dissipation in the Magnetotail
}

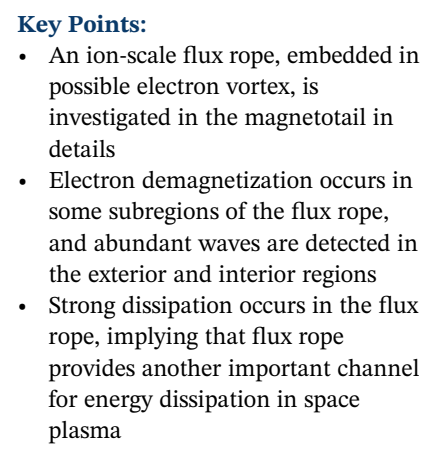

- An ion-scale flux rope, embedded in possible electron vortex, is investigated in the magnetotail in details

- Electron demagnetization occurs in some subregions of the flux rope, and abundant waves are detected in the exterior and interior regions

- Strong dissipation occurs in the flux rope, implying that flux rope provides another important channel for energy dissipation in space plasma

Correspondence to: S. Y. Huang,

shiyonghuang@whu.edu.cn

\begin{abstract}
S. Y. Huang ${ }^{1,2}$ (D) K. Jiang ${ }^{1}$ (D) Z. G. Yuan $^{1}$ (D), M. Zhou ${ }^{3}$ (D) F. Sahraoui ${ }^{4}$, H. S. Fu ${ }^{5}$ (D), X. H. Deng ${ }^{3}$ (D) Yu. V. Khotyaintsev ${ }^{6}$ (D) X. D. Yu' ${ }^{1}$ iD, L. H. He ${ }^{1}$, D. Deng ${ }^{1}$, C. J. Pollock ${ }^{7}$ (D), R. B. Torbert ${ }^{8}$ iD, and J. L. Burch ${ }^{9}$ iD

${ }^{1}$ School of Electronic Information, Wuhan University, Wuhan, China, ${ }^{2}$ Shandong Provincial Key Laboratory of Optical Astronomy and Solar-Terrestrial Environment, Weihai, China, ${ }^{3}$ Institute of Space Science and Technology, Nanchang University, Nanchang, China, ${ }^{4}$ Laboratoire de Physique des Plasmas, CNRS-Ecole Polytechnique-UPMC, Palaiseau, France, ${ }^{5}$ School of Space and Environment, Beihang University, Beijing, China, ${ }^{6}$ Swedish Institute of Space Physics, Uppsala, Sweden, ${ }^{7}$ NASA, Goddard Space Flight Center, Greenbelt, Maryland, USA, ${ }^{8}$ Institute for the Study of Earth, Oceans, and Space, University of New Hampshire, Durham, NH, USA, ${ }^{9}$ Southwest Research Institute, San Antonio, Texas, USA
\end{abstract}

\begin{abstract}
An ion-scale flux rope (FR), embedded in a high-speed electron flow (possibly an electron vortex), is investigated in the magnetotail using observations from the Magnetospheric Multiscale (MMS) spacecraft. Intense electric field and current and abundant waves are observed in the exterior and interior regions of the FR. Comparable parallel and perpendicular currents in the interior region imply that the FR has a non-force-free configuration. Electron demagnetization occurs in some subregions of the FR. It is surprising that strong dissipation $\left(J \times E^{\prime}\right.$ up to $\left.2,000 \mathrm{pW} / \mathrm{m}^{3}\right)$ occurs in the center of the FR without signatures of secondary reconnection or coalescence of two FRs, implying that FR may provide another important channel for energy dissipation in space plasmas. These features indicate that the observed FR is still highly dynamical, and hosts multiscale coupling processes, even though the FR has a very large scale and is far away from the reconnection site.
\end{abstract}

Plain Language Summary Flux ropes, 3-D helical magnetic structures, in which magnetic field lines twist with each other, play an important role in the macroscopic and microscopic physical process during magnetic reconnection. Most of previous studies focused on the flux ropes in the reconnection region. However, some physical process inside macroscopic flux ropes far away from the reconnection site in the magnetotail is still unclear due to the lack of high time resolution data. In this letter, thanks to the unprecedented high time resolution data of the Magnetospheric Multiscale (MMS) mission, we report an ion-scale flux rope and study its dynamics. Our observations demonstrate that the observed flux rope is still highly dynamical, and hosting multiscale coupling processes and strong energy dissipation, even though the flux rope has very large scale and is far away from the reconnection site.

\section{Introduction}

Magnetic reconnection is one of the most important physical processes in space, astrophysical, and laboratory plasmas, which can effectively convert magnetic energy into plasma kinetic and thermal energy in a bursty way, changing the topology of the magnetic field. Flux ropes (FRs) are 3-D helical magnetic structures in which magnetic field lines twist with each other and a strong core field generally exists; FRs can be formed by single X-line or multiple X-line magnetic reconnection (e.g., Deng et al., 2004; Fu et al., 2015, 2016, 2017; Huang, Pang, et al., 2014; Huang, Vaivads, et al., 2012; Huang, Zhou, et al., 2014; Huang, Zhou, et al., 2015; Lee \& Fu, 1985; Nakamura \& Scholer, 2000; Slavin et al., 2003; Zhou et al., 2012, 2017). FRs have been frequently observed in the Earth's magnetosphere (e.g., Chen et al., 2008; Huang, Retino, et al., 2016; Huang, Sahraoui, et al, 2016; Huang, Vaivads, et al., 2012; Retinò et al., 2008; Slavin et al., 2003; Wang et al., 2010; Zhao, Wang, Lu, et al., 2016; Zong et al., 2004). FRs can have multiscale structures or multiple layers (e.g., Huang, Retino, et al., 2016; Hwang et al., 2016), and may show different features in the electron velocity distributions (e.g., Zhong et al., 2013) and different wave properties in different subregions (e.g., Huang, Retino, et al., 2016; Khotyaintsev et al., 2010; Wang, Lu, Nakamura, Huang, Du, et al., 2016). 
It is believed that FRs play an important role in the macroscopic and microscopic physical processes occurring during magnetic reconnection, such as modulation of reconnection rates and electron accelerations (e.g., Chen et al., 2008; Daughton et al., 2006, 2011; Drake et al., 2006; Fu, Cao, et al., 2013; Fu, Khotyaintsev, et al., 2013; Fujimoto, 2017). Simulations have shown that the electrons can be accelerated by the contracting of FRs via the Fermi acceleration mechanism (Drake et al., 2006; Fu et al., 2006), by the reconnection electric field in the secondary FRs (Oka, Fujimoto, et al., 2010), and by the coalescence of FRs (Oka, Phan, et al., 2010; Pritchett, 2008; Zhou et al., 2014) in the reconnection region. The enhancement of energetic electrons has been observed inside the FRs (Chen et al., 2008; Huang, Vaivads, et al., 2012; Retinò et al., 2008; Wang et al., 2010). The observational evidence of the coalescence of FRs has been shown using high-resolution data from the Cluster and MMS missions (Wang, Lu, Nakamura, Huang, Li, et al., 2016; Zhao, Wang, \& Du, 2016; Zhou et al., 2017). In one particular event, an ongoing reconnection site (i.e., electron diffusion region) with strong energy dissipation was directly detected in the interaction region of two macroscopic FRs at the magnetopause (Zhou et al., 2017). However, some physical processes occurring inside macroscopic FRs far away from the reconnection site in the magnetotail are still unclear due to the lack of high time resolution data. In this paper, we use the unprecedented high-resolution data from NASA's MMS mission to investigate FRs in the near-Earth magnetotail, and try to understand the physical process occurring inside the FRs.

\section{MMS Observations}

The magnetic field data recorded by the Fluxgate Magnetometer (Russell et al., 2016) and Search-Coil Magnetometer (Le Contel et al., 2016), the electric field data collected by the Electric Double Probe (Ergun et al., 2016; Lindqvist et al., 2016), and the 3-D particle distribution functions and the plasma moments measured by the Fast Plasma Instrument (Pollock et al., 2016) onboard the MMS spacecraft are used in this study.

Figure 1 displays an overview when the MMS encountered a series of FRs and crossed the current sheet repeatedly. The four MMS spacecraft formed a tetrahedron with the maximum separation $\sim 26 \mathrm{~km}$. The data recorded by four MMS spacecraft are nearly identical at such small separation, so we only present MMS1's data here. During the interval of interest, MMS spacecraft were located at $[-17.7,3.7,2.4] R_{E}$ in GSM coordinates $\left(R_{E}\right.$ is the Earth's radius). From the typical ion spectrogram (Figure 1a), plasma density $\left(N_{i} \sim 0.4 \mathrm{~cm}^{3}\right.$; Figure 1d), high ion temperature ( $\sim$ several keV; Figure 1e), and large plasma $\beta$ (up to $10^{3}$; Figure 1f), one can infer that the MMS were in the magnetotail plasma sheet (Cao et al., 2006). MMS initially stayed in the southern hemisphere and then crossed the current sheet 6 times and finally went back to the southern hemisphere (Figure 1b). $V_{i x}$ is generally positive and has maximum values of more than $800 \mathrm{~km} / \mathrm{s}$ around 00:56:20 UT during this interval, implying that the MMS spacecraft were embedded in earthward plasma flow. With the bipolar variations in $B_{z}$ component (from negative to positive), peaks in $B_{x}$ or $B_{y}$ component, and amplitude of magnetic field $B_{t}$ in the earthward flow, one can identify at least five earthward moving FRs (as indicated by the red dashed circles in Figure 1b). There are enhancements of ion temperature and density in the FRs. The third FR indicated by two black dashed lines will be discussed below in detail.

Figure 2 shows the details of the third FR in LMN coordinates $(L=[0.98,-0.13,-0.15], M=[0.15,0.97$, $0.19], N=[0.13,-0.21,0.97]$ in GSM coordinates). The LMN coordinates are determined by minimum variance analysis (MVA; Sonnerup \& Scheible, 1998; from 00:56:25.9050 UT to 00:56:28.3152 UT). The angle between $N$ direction of LMN and normal direction ([-0.05, 0.30, -0.95]) determined by timing analysis is $7^{\circ}$; thus, the results in LMN coordinates are reliable. The MMS spacecraft stayed off-equator in the southern hemisphere of the plasma sheet since the $B_{L}$ component is negative (up to $-28 \mathrm{nT}$ ), comparable to $B_{t}$. When $B_{N}$ changes from negative to positive (Figure $2 \mathrm{~d}$ ) in the earthward flow ( $V_{i L}>0$ in Figure 2e), both $B_{t}$ and $B_{L}$ have a peak (Figures $2 \mathrm{a}$ and $2 \mathrm{~b}$ ). These signatures are all unambiguous features of an earthward moving FR. The scale of the FR is estimated to be $2,210 \pm 230 \mathrm{~km}$ from timing analysis, that is, $\sim 6.1 \pm 0.6 \lambda_{i}\left(\lambda_{i} \sim 360 \mathrm{~km}\right.$ is ion inertial length based on the average asymptotic plasma parameters $|B| \sim 22 \mathrm{nT}, N_{i}=N_{e} \sim 0.4 \mathrm{~cm}^{3}$ ), implying that this FR is an ion-scale structure. Different features of the plasma observations can be used to distinguish the exterior and interior regions of this FR (see bars at the top of Figure 2a). Accompanying the earthward flow, $V_{i M}$ increases inside the FR $(\sim 00 \mathrm{~km} / \mathrm{s}$ in Figure $2 \mathrm{f})$, indicating that a high-speed duskward component of ion flow is present in the interior region (BC) of the FR. It is worth noting that strong electron 


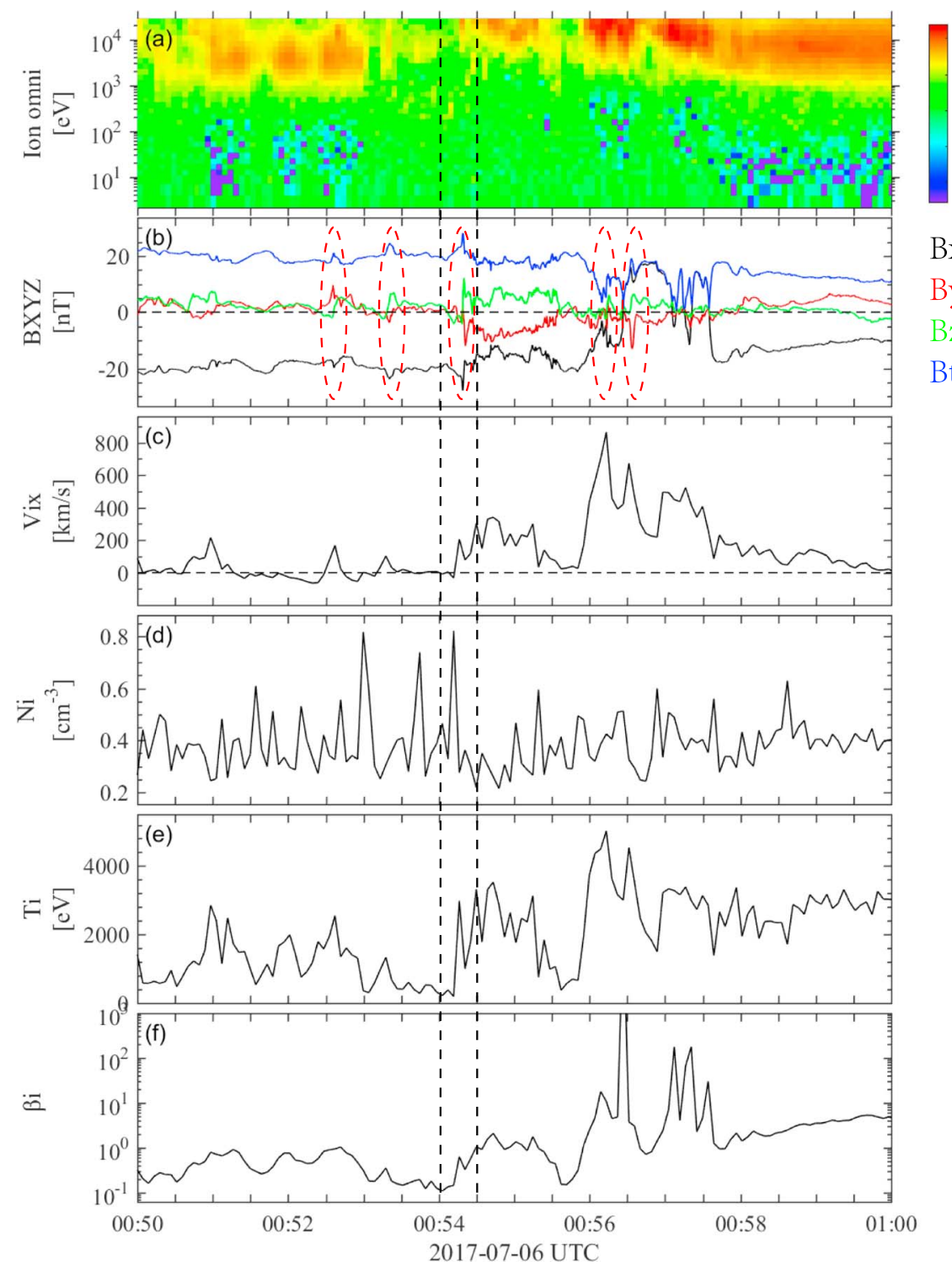

Figure 1. Overview of MMS1 observations in GSM coordinates: (a) ion differential energy flux, (b) magnetic field, ion (c) velocity, (d) density, (e) temperature, and (f) plasma $\beta$. The red dashed circles mark the observed FRs and the black dashed lines mark the interval of the FR investigated in this work.

flow is detected in the exterior region of FR: $V_{e L}$ is up to $-14,000 \mathrm{~km} / \mathrm{s}, V_{e M}$ is $\sim-4,000 \mathrm{~km} / \mathrm{s}$, and $V_{e N}$ is $\sim-3,000 \mathrm{~km} / \mathrm{s}$ (Figures $2 \mathrm{~h}-2 \mathrm{j}$ ) from 00:54:13.5 UT to 00:54:16 UT in the inbound exterior region (AB) prior to entering the FR; while $V_{e L}$ is up to $-7,500 \mathrm{~km} / \mathrm{s}, V_{e M}$ is $\sim-5,000 \mathrm{~km} / \mathrm{s}$, and $V_{e N}$ is $\sim 3,000 \mathrm{~km} / \mathrm{s}$ from 00:54:20.5 UT to 00:54:24 UT in the outbound exterior region (CD) of the FR. Thus, $V_{e L}$ stays negative but $V_{e N}$ changes from negative to positive in the exterior region, which indicates that the FR may be surrounded by an electron vortex (as illustrated in Figure $2 \mathrm{~m}$ ). However, the electron flow is smaller in the interior region than in the exterior region except for one peak in $V_{e N}$ (up to $2,000 \mathrm{~km} / \mathrm{s}$ ) in the center of the FR that is marked by the cyan bar in Figure 2. Both electron temperature and density increase in the FR, and an obvious electron temperature anisotropy is detected in the center of the FR $\left(T_{e \| l}>T_{e \perp}\right.$ in Figure 2k).

Figure 3 presents the electric field, current density, and $J \times E^{\prime}$ associated with the FR. The electric field is very intense in the exterior and interior regions of the FR, especially reaching values of up to $150 \mathrm{mV} / \mathrm{m}$ in the center of FR (Figures 3b-3d), while the electric field is small and close to zero far away from the FR 

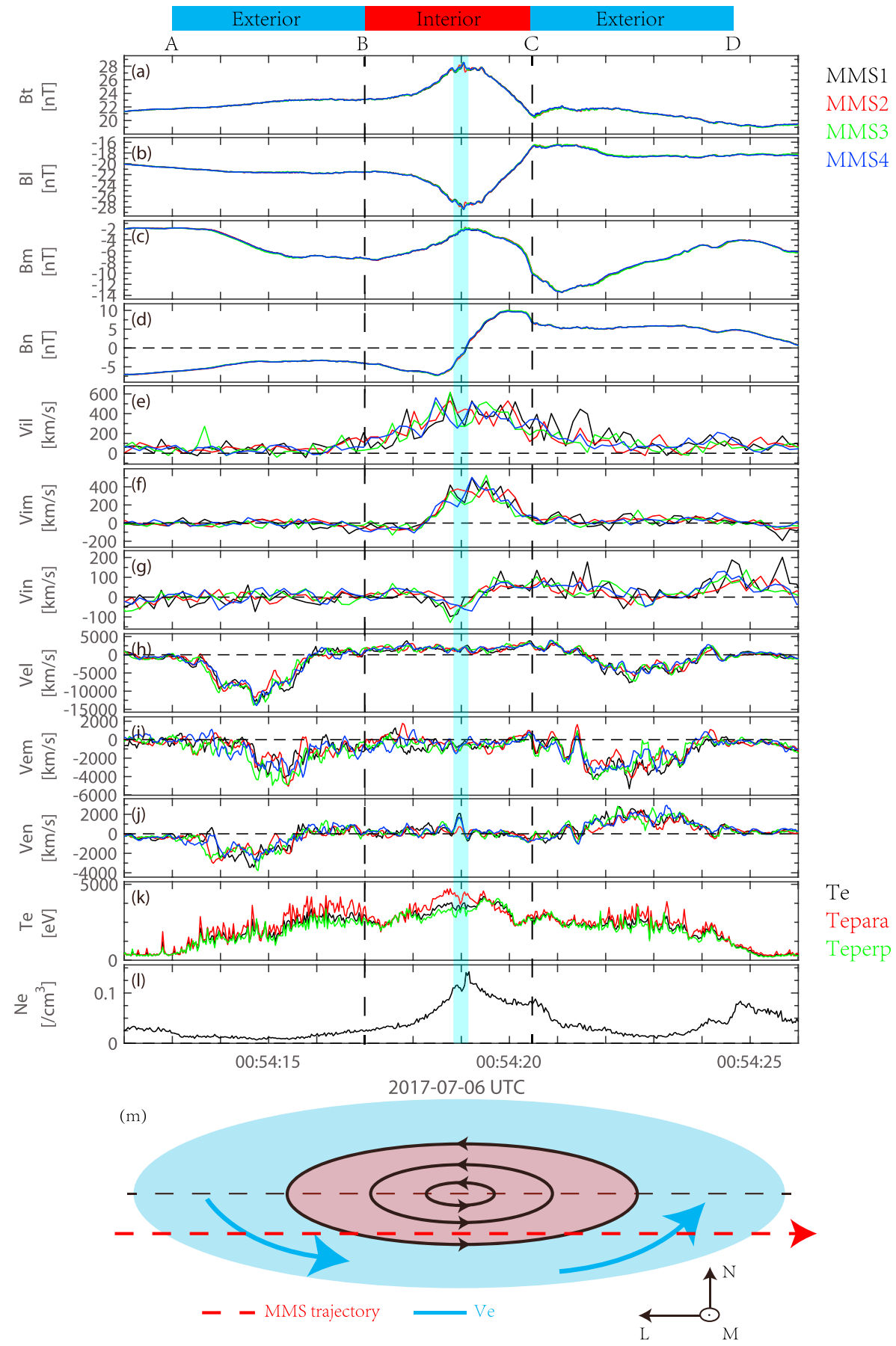

Figure 2. Detailed observations of the third FR in Figure 1. (a-j) All four MMS spacecraft observations. (a-d) Magnetic field; $L, M, N$ components of (e-g) ion velocity and (h-j) electron velocity; (k) electron temperature and (l) density recorded by MMS1; and (m) cartoon diagram of the FR with the MMS trajectory and electron flow. The cyan and red shadow indicate the exterior and interior regions of the FR. The vertical black dashed lines mark the FR and the cyan shade marks the interval of significant dissipation. The color bars at the top of the figure indicate the exterior region (blue) and interior region (red) of the FR, unless specified indicated.

(before 00:54:13 UT and after 00:54:24 UT). $V_{i} \times B$ and $V_{e} \times B$ are also calculated to compare with electric field $E$ in Figures 3b-3d. It is interesting that $V_{i} \times B$ does not agree with the three components of electric field during the entire interval, which occurs because the FPI underestimates the ions in the plasma sheet since its energy range is limited to $<30 \mathrm{keV}$ (Pollock et al., 2016) while ions with energy $>30 \mathrm{keV}$ are 


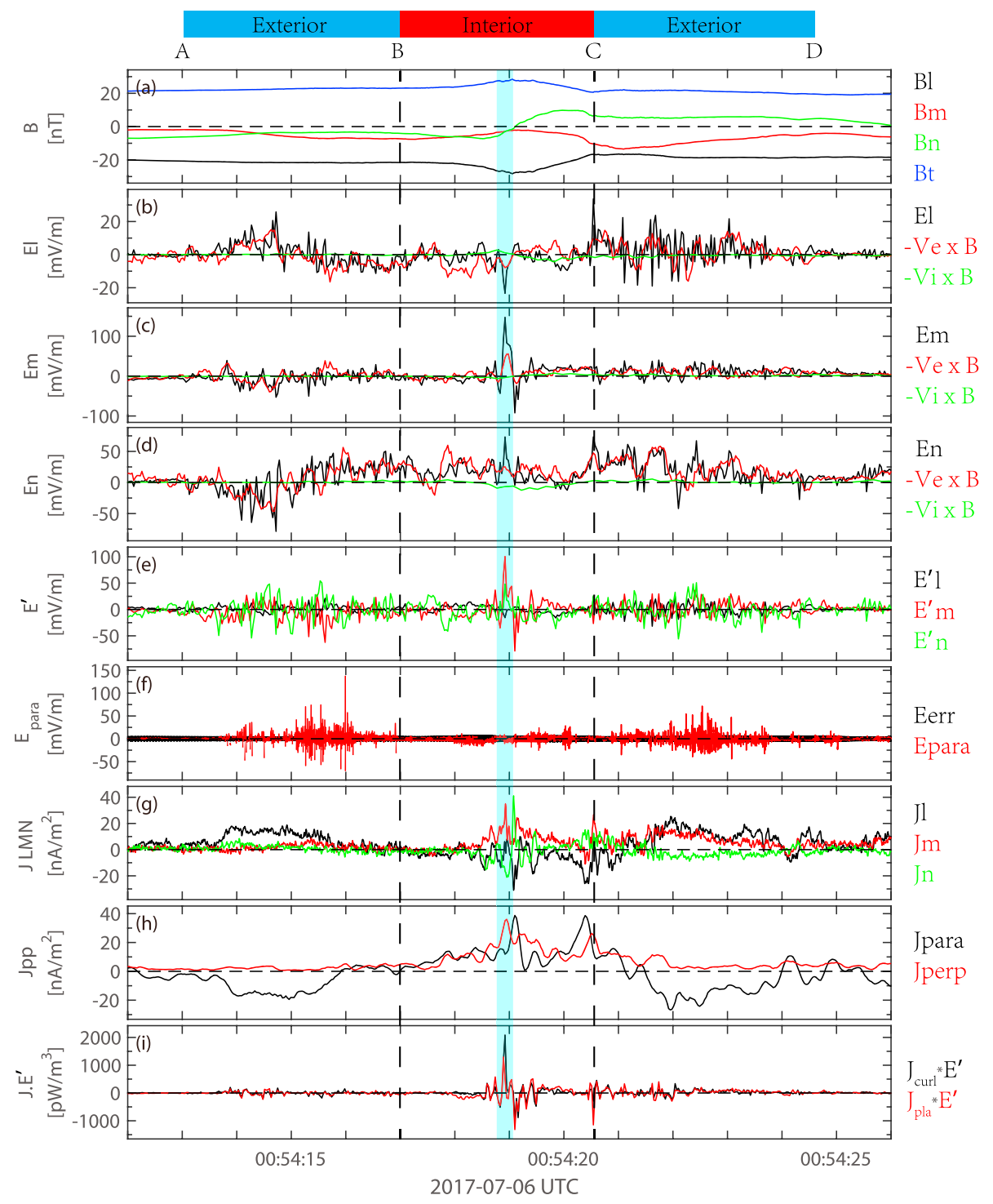

Figure 3. Detailed observations of the third FR. (a) Magnetic field; (b-d) $L, M$, and $N$ components of electric field, $-V_{e} \times B$ and $-V_{i} \times B$, respectively; (e) $E^{\prime}=E+V_{e} \times B$; (f) parallel electric field and error; (g) three components of current $J$ calculated by curlometer method; (h) parallel and perpendicular currents; and (i) the dissipation term where $J_{\text {curl }} \times E^{\prime}$ calculated from curlometer method, and $J_{\text {pla }} \times E^{\prime}$ calculated from plasma data.

dominant in the entire plasma sheet at this time (Figure 1a). Fortunately, the FPI can accurately measure the entire electrons population. One can see that there are some differences between electric field and $V_{e} \times B$ in short time periods in the FR. To clearly identify such differences, $E^{\prime}=E+V_{e} \times B$ is calculated and shown in Figure 3e. The nonzero $E^{\prime}$ in the FR implies that there are some filaments with decoupling between the electrons and magnetic field lines. The strongest nonzero peak of $E^{\prime}$ occurs in the center of the FR (marked by the cyan bar), up to $100 \mathrm{mV} / \mathrm{m}$, indicating that the electrons can be demagnetized even in an ion-scale FR. The parallel electric field $E_{||}$has large fluctuations with $\sim 50 \mathrm{mV} / \mathrm{m}$ amplitude in the exterior region, but smaller fluctuations with $\sim 25 \mathrm{mV} / \mathrm{m}$ amplitude in the interior region. Intense currents calculated by the curlometer method are observed both in the exterior and interior regions of FR (Figure 3g). Parallel current is dominant in the exterior region, while it becomes comparable to the perpendicular one in the interior region (Figure 3h), implying that the FR has a non-force-free configuration. Different polarities of parallel current outside and inside the FR can cause magnetic dip in the interfaces between exterior and interior regions (see $B_{t}$ in Figure 3a). There are several peaks of 

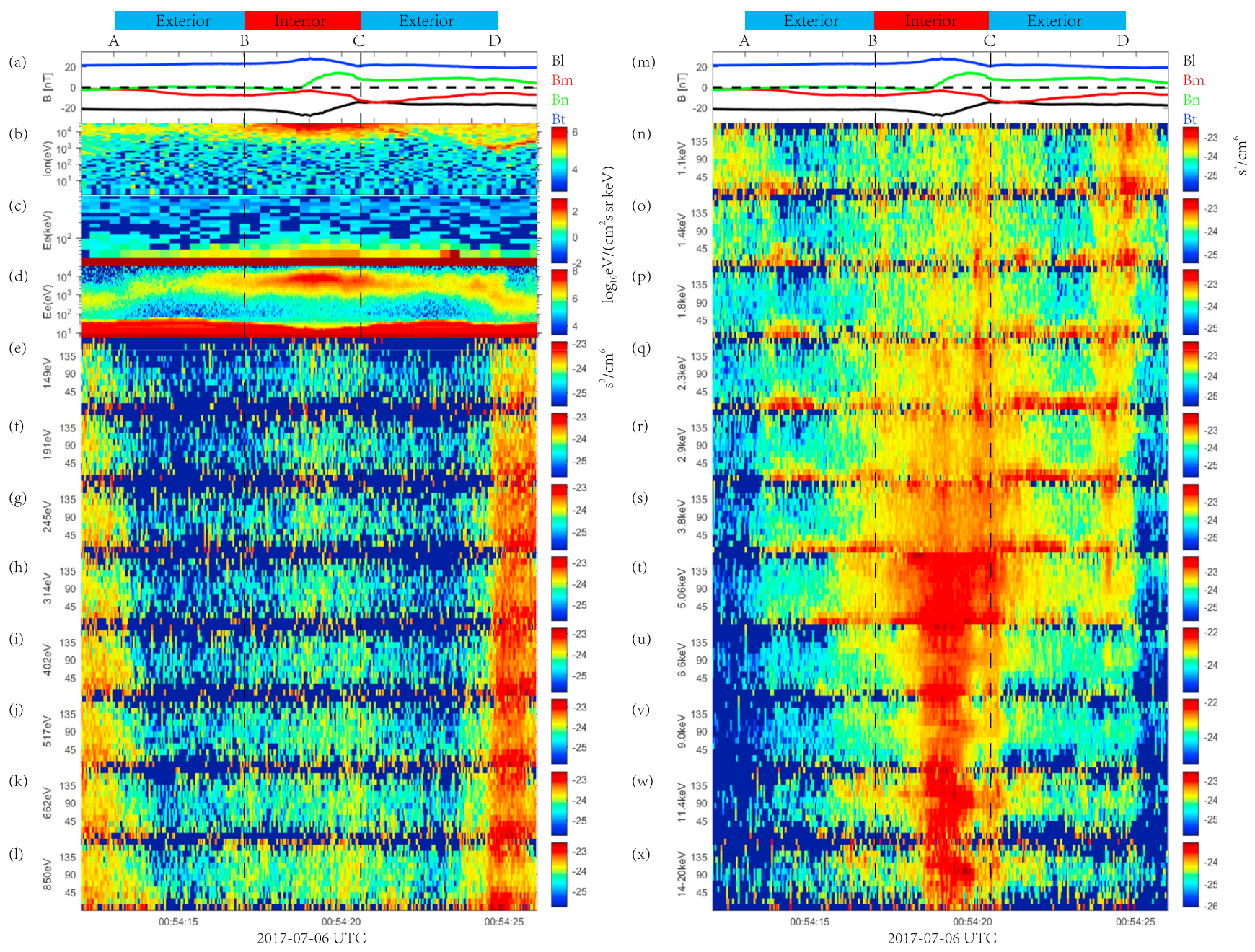

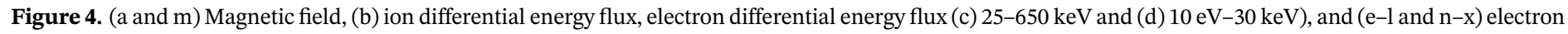
pitch angle distributions of different energy levels.

current density in the FR, implying the existence of current filaments (e.g., Fu et al., 2017). The energy dissipation parameter $(J \times E)$ derived from both the curlometer method and plasma method are shown in Figure 3i. The results from the two methods are similar. One can see that strongest energy dissipation $\left(J \times E^{\prime}\right.$ up to $2,000 \mathrm{pW} / \mathrm{m}^{3}$; energy transfer from magnetic field to the particles) occurs in the center of the FR where intense nonzero $E^{\prime}$ and intense current exist, which is even stronger than the dissipation in the magnetotail reconnection site (Huang et al., 2018). Such measurements support the proposition that energy dissipation occurs at O-lines, but not X-lines in the reconnection region by Fu et al. (2017).

Figure 4 displays ion and electron differential energy fluxes and electron pitch angle distributions. Significant increase of ion fluxes (from several $\mathrm{keV}$ to $>30 \mathrm{keV}$; Figure 4b), and enhancements of electron fluxes (from several $\mathrm{keV}$ to tens of $\mathrm{keV}$ in Figure 4d, and up to $100 \mathrm{keV}$ in Figure 4c) are observed in the FR. Due to the low quality of the FPI data for electrons with energy below $100 \mathrm{eV}$, Figures $4 \mathrm{e}-4 \mathrm{x}$ only show the pitch angle distributions of the electrons with the energy from $149 \mathrm{eV}$ to $20 \mathrm{keV}$. It can be clearly seen that a parallel electron beam is present at the energy levels from $\sim 1$ to $\sim 5 \mathrm{keV}$ in the exterior region and a field-aligned electron beam also exists at the energy levels from $\sim 2$ to $20 \mathrm{keV}$ in the interior region. In addition, there are enhancements of phase space densities at $\sim 90^{\circ}$ at energy above $5 \mathrm{keV}$ in the interior region, implying that these electrons may be trapped in the FR. Such distributions of electrons in the observed FR may lead to plasma instabilities and cause the generations of waves. Figure 5 illustrates intense wave activity from $f_{\text {ci }}$ (proton gyrofrequency) to $f_{\text {ce }}$ (electron gyrofrequency). There are at least three different types of waves associated with the FR. The first type is intermittent whistler mode waves with enhancements of 

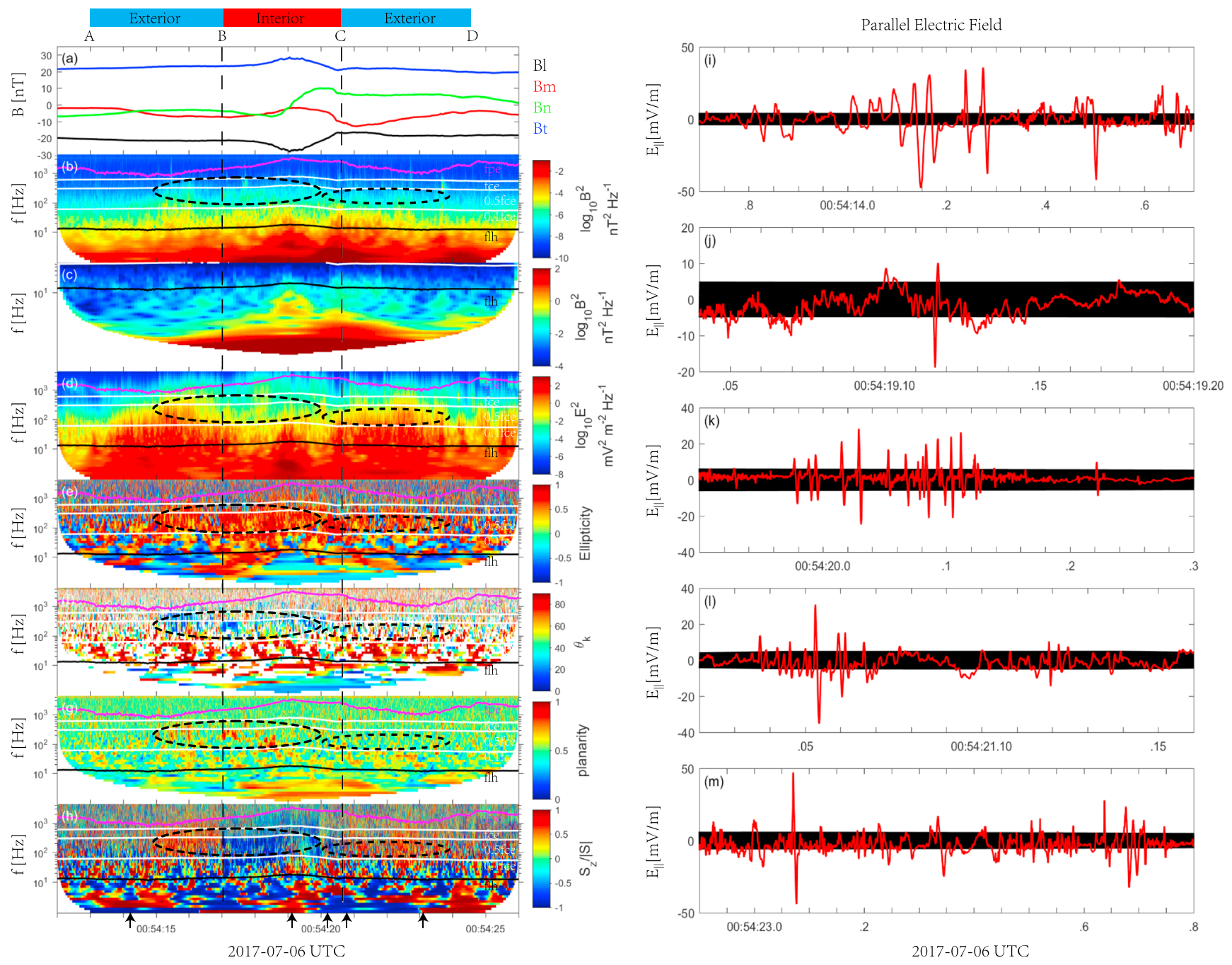

Figure 5. The wave analysis of MMS1. (a) Magnetic field, the magnetic spectra (b) (SCM; 8,192 Hz), (c) (FGM; $128 \mathrm{~Hz}$ ), (d) the electric spectra (EDP; 8,192 Hz), (e) ellipticities (red: right-hand polarization; blue: left-hand polarization), (f) propagation angles, $\theta_{k}$, (g) planarity of polarization, (h) Poynting flux, and (i-m) parallel electric field during the five intervals marked by arrows below (h). The black dashed circles mark the whistler waves. The magenta, white, and black horizontal lines in (b)-(h) represent electron plasma frequency $\left(f_{\mathrm{pe}}\right)$, electron gyrofrequency $\left(f_{\mathrm{ce}}, 0.5 f_{\mathrm{ce}}, 0.1 f_{\mathrm{ce}}\right.$; from top to bottom), and lower hybrid frequency $\left(f_{\text {lh }}\right)$, respectively.

electromagnetic power spectral densities between $0.1 f_{\text {ce }}$ and $f_{\text {ce }}$ (Figures $5 \mathrm{~b}$ and $5 \mathrm{~d}$ ), right-hand polarization (red in Figure 5e), and quasi-parallel propagation (blue in Figure 5f), both in the exterior and interior regions of the FR (as marked by black dashed circles in Figure 5). The whistlers propagate parallel to the magnetic field in the exterior region, while they propagate antiparallel to the magnetic field in the interior region (Figure 5h). The second type is lower hybrid waves both in the exterior region and interior region, which appears as enhancements of electromagnetic power spectral densities around the lower hybrid frequency $\left(f_{\mathrm{lh}}\right)$. The third type is an electrostatic solitary wave (Omura et al., 1996). Figures 5i-5m show a series of bipolar signatures in the parallel electric field with a duration of tens of milliseconds and amplitudes of $15 \sim 50 \mathrm{mV} / \mathrm{m}$ in the exterior and interior regions of the FR (as marked below Figure 5h by black arrows), which are consistent with typical features of electrostatic solitary waves.

\section{Discussion}

There are many dynamical instabilities, different types of waves, and multiscale processes occurring in reconnection regions (e.g., Cao et al., 2017; Huang et al., 2010; Huang, Vaivads, et al., 2012; Vaivads et al., 2006; Zhou et al., 2014). Electrostatic and electromagnetic lower hybrid frequency range waves are detected 
and are associated with FRs in the magnetotail ion diffusion region (Wang, Lu, Nakamura, Huang, Li, et al., 2016). One ion-scale FR, away far from the reconnection site, was identified in the turbulent magnetosheath for the first time (Huang, Retino, et al., 2016). Electrostatic solitary waves and strong lower hybrid drift waves are detected inside the FR, while whistler waves, weak electromagnetic lower hybrid drift waves, and strong broadband electrostatic noise are detected at the edges of FR (Huang, Retino, et al., 2016). In the present study, several types of waves are detected associated with the FR in the magnetotail. Intermittent whistler emissions (electron-scale), electrostatic solitary waves (electron-scale), and lower hybrid waves are observed both in the exterior and interior regions of the ion-scale FR; however, this FR is not observed in or near the reconnection site. Thus, the observations of the waves associated with the FRs indicate that highly dynamical and multiscale coupled processes can also occur in the FRs even far away from the reconnection site.

The intermittent whistler waves are observed in the exterior and interior regions of the FR in our event. The whistlers in the exterior region propagate parallel to the magnetic field, while the whistlers in the interior region propagate antiparallel to the magnetic field. This may indicate that the observed whistlers have different sources. Recently, kinetic simulations (Fujimoto, 2014; Fujimoto \& Sydora, 2008; Huang, Fu, et al., 2016; Huang et al., 2017) have confirmed that there are two different types of whistler waves in the reconnection region: one is triggered in the equatorial plane of the pileup region and propagates downstream and the other is generated at the separatrix region and propagates toward the $\mathrm{X}$-line. Thus, one may deduce that the whistlers in the exterior region are produced at the separatrix region and propagate toward the X-line that is located at the right side of the observed FR; the whistlers in the interior region are generated at the equatorial plane of the pileup region of the X-line. Both whistlers pass through the spacecraft after they are generated.

Strong energy dissipation typically occurs in the electron diffusion region during magnetic reconnection (e.g., Burch et al., 2016; Zhou et al., 2017) and at dipolarization fronts (Huang, Zhou, et al., 2012; Huang, Fu, et al., 2015; Liu et al., 2018). Fu et al. (2017) have found strongest energy dissipation at O-lines (can be treated as FRs) in one magnetic reconnection diffusion region due to the strongest currents and kinetic turbulence there. However, as reported here, MMS detected strongest energy dissipation in the center of a largescale FR that is far away from the reconnection site. In addition, there is no direct evidence for the existence of coalescence of two FRs or secondary reconnection in the FR. Only one clue is the density dip in the core region with the strongest currents that can be the consequence of the coalescence of two FRs (Retinò et al., 2008), indicating that strong dissipation may be caused by the coalescence of FRs. Our observations imply that the FR provides an important channel for the energy dissipation in space plasmas.

\section{Summary}

We report an ion-scale FR in the magnetotail observed by MMS spacecraft. This FR, surrounded by highspeed electron flow (possibly an electron vortex), is embedded in an earthward plasma flow. Both electron density and temperature increase in the FR, and intense currents are found in the interior and exterior regions of the FR. Parallel and perpendicular currents are comparable in the interior region, implying that the FR has a non-force-free configuration. There are strong electric fields in the FR and large parallel electric fields in the exterior region. The electric field in the electron frame $\left(E+V_{e} \times B\right.$ ) is nonzero (up to $\sim 100 \mathrm{mV} / \mathrm{m}$ ) in the subregions of the FR, especially in the center, indicating that the electrons are decoupled from magnetic field lines. Wave activity, including whistler waves, lower hybrid waves, and electrostatic solitary waves, is observed in the FR, implying that multiscale coupling processes occur in the large-scale FR. Strongest dissipation is detected in the center of FR, indicating that the FR is still highly dynamical even though it has a very large scale and is far away from the reconnection site.

Acknowledgments
We thank the entire MMS team and instrument leads for the data access and support. This work was supported by the National Natural Science Foundation of China (41674161, 41874191). S.Y.H. acknowledges the project supported by the Specialized Research Fund for Shandong Provincial Key Laboratory and the support by Young Elite Scientists Sponsorship Program by CAST (2017QNRC001). MMS data are publicly available from the MMS Science Data Center at http:// lasp.colorado.edu/mms/sdc/.

\section{References}

Burch, J. L., Torbert, R. B., Phan, T. D., Chen, L. J., Moore, T. E., Ergun, R. E., et al. (2016). Electron-scale measurements of magnetic reconnection in space. Science, 352(6290), aaf2939. https://doi.org/10.1126/science.aaf2939

Cao, D., Fu, H. S., Cao, J. B., Wang, T. Y., Graham, D. B., Chen, Z. Z., et al. (2017). MMS observations of whistler waves in electron diffusion region. Geophysical Research Letters, 44, 3954-3962. https://doi.org/10.1002/2017GL072703

Cao, J. B., Ma, Y. D., Parks, G., Reme, H., Dandouras, I., Nakamura, R., et al. (2006). Joint observations by Cluster satellites of bursty bulk flows in the magnetotail. Journal of Geophysical Research, 111, A04206. https://doi.org/10.1029/2005JA011322

Chen, L. J., Bhattacharjee, A., Puhl-Quinn, P. A., Yang, H., Bessho, N., Imada, S., et al. (2008). Observation of energetic electrons within magnetic islands. Nature Physics, 4(1), 19-23. https://doi.org/10.1038/nphys777 
Contel, O. L., Leroy, P., Roux, A., Coillot, C., Alison, D., Bouabdellah, A., et al. (2016). The search-coil magnetometer for MMS. Space Science Reviews, 199(1-4), 257-282.

Daughton, W., Roytershteyn, V., Karimabadi, H., Yin, L., Albright, B. J., Bergen, B., \& Bowers, K. J. (2011). Role of electron physics in the development of turbulent magnetic reconnection in collisionless plasmas. Nature Physics, 7(7), 539-542. https://doi.org/10.1038/ nphys1965

Daughton, W., Scudder, J., \& Karimabadi, H. (2006). Fully kinetic simulations of undriven magnetic reconnection with open boundary conditions. Physics of Plasmas, 13(7), 072101. https://doi.org/10.1063/1.2218817

Deng, X. H., Matsumoto, H., Kojima, H., Mukai, T., Anderson, R. R., Baumjohann, W., \& Nakamura, R. (2004). Geotail encounter with reconnection diffusion region in the Earth's magnetotail: Evidence of multiple X lines collisionless reconnection? Journal of Geophysical Research, 109, A05206. https://doi.org/10.1029/2003JA010031

Drake, J. F., Swisdak, M., Che, H., \& Shay, M. A. (2006). Electron acceleration from contracting magnetic islands during reconnection. Nature, 443(7111), 553-556. https://doi.org/10.1038/nature05116

Ergun, R. E., Tucker, S., Westfall, J., Goodrich, K. A., Malaspina, D. M., Summers, D., et al. (2016). The axial double probe and fields signal processing for the mms mission. Space Science Reviews, 199(1-4), 167-188. https://doi.org/10.1007/s11214-014-0115-x

Fu, H. S., Cao, J. B., Khotyaintsev, Y. V., Sitnov, M. I., Runov, A., Fu, S. Y., et al. (2013). Dipolarization fronts as a consequence of transient reconnection: In situ evidence. Geophysical Research Letters, 40, 6023-6027. https://doi.org/10.1002/2013GL058620

Fu, H. S., Cao, J. B., Vaivads, A., Khotyaintsev, Y. V., Andre, M., Dunlop, M., et al. (2016). Identifying magnetic reconnection events using the FOTE method. Journal of Geophysical Research: Space Physics, 121, 1263-1272. https://doi.org/10.1002/2015JA021701

Fu, H. S., Khotyaintsev, Y. V., Vaivads, A., Retinò, A., \& André, M. (2013). Energetic electron acceleration by unsteady magnetic reconnection. Nature Physics, 9(7), 426-430. https://doi.org/10.1038/nphys2664

Fu, H. S., Vaivads, A., Khotyaintsev, Y. V., André, M., Cao, J. B., Olshevsky, V., et al. (2017). Intermittent energy dissipation by turbulent reconnection. Geophysical Research Letters, 44, 37-43. https://doi.org/10.1002/2016GL071787

Fu, H. S., Vaivads, A., Khotyaintsev, Y. V., Olshevsky, V., André, M., Cao, J. B., et al. (2015). How to find magnetic nulls and reconstruct field topology with MMS data? Journal of Geophysical Research: Space Physics, 120, 3758-3782. https://doi.org/10.1002/2015JA021082

Fu, X. R., Lu, Q. M., \& Wang, S. (2006). The process of electron acceleration during collisionless magnetic reconnection. Physics of Plasmas, 13(1), 012309. https://doi.org/10.1063/1.2164808

Fujimoto, K. (2014). Wave activities in separatrix regions of magnetic reconnection. Geophysical Research Letters, 41, 2721-2728. https:// doi.org/10.1002/2014GL059893

Fujimoto, K. (2017). Bursty emission of whistler waves in association with plasmoid collision. Annales Geophysicae, 35, 885-892. https:// doi.org/10.5194/angeo-35-885-2017

Fujimoto, K., \& Sydora, R. D. (2008). Whistler waves associated with magnetic reconnection. Geophysical Research Letters, 35, L19112. https://doi.org/10.1029/2008GL035201

Huang, S., Pang, Y., Yuan, Z., Deng, X., He, J., Zhou, M., et al. (2014). Observation of directional change of core field inside flux ropes within one reconnection diffusion region in the Earth's magnetotail. Chinese Science Bulletin. https://doi.org/10.1007/s11434-0140583-0

Huang, S. Y., Fu, H. S., Yuan, Z. G., Vaivads, A., Khotyaintsev, Y. V., Retino, A., et al. (2016). Two types of whistler waves in the hall reconnection region. Journal of Geophysical Research: Space Physics, 121, 6639-6646. https://doi.org/10.1002/2016JA022650

Huang, S. Y., Fu, H. S., Yuan, Z. G., Zhou, M., Fu, S., Deng, X. H., et al. (2015). Electromagnetic energy conversion at dipolarization fronts: Multispacecraft results. Journal of Geophysical Research: Space Physics, 120, 4496-4502. https://doi.org/10.1002/2015JA021083

Huang, S. Y., Jiang, K., Yuan, Z. G., Sahraoui, F., He, L. H., Zhou, M., et al. (2018). Observations of the electron jet generated by secondary reconnection in the terrestrial magnetotail. The Astrophysical Journal, 862, 144. https://doi.org/10.3847/1538-4357/aacd4c

Huang, S. Y., Retino, A., Phan, T. D., Daughton, W., Vaivads, A., Karimabadi, H., et al. (2016). In situ observations of flux rope at the separatrix region of magnetic reconnection. Journal of Geophysical Research: Space Physics, 121, 205-213. https://doi.org/10.1002/ 2015JA021468

Huang, S. Y., Sahraoui, F., Retino, A., le Contel, O., Yuan, Z. G., Chasapis, A., et al. (2016). MMS observations of ion-scale magnetic island in the magnetosheath turbulent plasma. Geophysical Research Letters, 43, 7850-7858. https://doi.org/10.1002/2016GL070033

Huang, S. Y., Vaivads, A., Khotyaintsev, Y. V., Zhou, M., Fu, H. S., Retinò, A., et al. (2012). Electron acceleration in the reconnection diffusion region: Cluster observations. Geophysical Research Letters, 39, L11103. https://doi.org/10.1029/2012GL051946

Huang, S. Y., Yuan, Z. G., Sahraoui, F., Fu, H. S., Pang, Y., Zhou, M., et al. (2017). Occurrence rate of whistler waves in the magnetotail reconnection region. Journal of Geophysical Research: Space Physics, 122, 7188-7196. https://doi.org/10.1002/2016JA023670

Huang, S. Y., Zhou, M., Deng, X. H., Yuan, Z. G., Pang, Y., Wei, Q., et al. (2012). Kinetic structure and wave properties associated with sharp dipolarization front observed by Cluster. Annales de Geophysique, 30(1), 97-107. https://doi.org/10.5194/angeo-30-97-2012

Huang, S. Y., Zhou, M., Sahraoui, F., Deng, X. H., Pang, Y., Yuan, Z. G., et al. (2010). Wave properties in the magnetic reconnection diffusion region with high $\beta$ : Application of the k-filtering method to cluster multispacecraft data. Journal of Geophysical Research, 115, A12211. https://doi.org/10.1029/2010JA015335

Huang, S. Y., Zhou, M., Yuan, Z. G., Deng, X. H., Sahraoui, F., Pang, Y., \& Fu, S. (2014). Kinetic simulations of electric field structure within magnetic island during magnetic reconnection and their applications to the satellite observations. Journal of Geophysical Research: Space Physics, 119, 7402-7412. https://doi.org/10.1002/2014JA020054

Huang, S. Y., Zhou, M., Yuan, Z. G., Fu, H. S., He, J. S., Sahraoui, F., et al. (2015). Kinetic simulations of secondary reconnection in the reconnection jet. Journal of Geophysical Research: Space Physics, 120, 6188-6198. https://doi.org/10.1002/2014JA020969

Hwang, K.-J., Sibeck, D. G., Giles, B. L., Pollock, C. J., Gershman, D., Avanov, L., et al. (2016). The substructure of a flux transfer event observed by the MMS spacecraft. Geophysical Research Letters, 43, 9434-9443. https://doi.org/10.1002/2016GL070934

Khotyaintsev, Y. V., Vaivads, A., André, M., Fujimoto, M., Retinò, A., \& Owen, C. J. (2010). Observations of slow electron holes at a magnetic reconnection site. Physical Review Letters, 105(16), 165002. https://doi.org/10.1103/PhysRevLett.105.165002

Lee, L. C., \& Fu, Z. F. (1985). A theory of magnetic flux transfer at the Earth 's magnetopause. Geophysical Research Letters, 12, $105-108$. https://doi.org/10.1029/GL012i002p00105

Lindqvist, P.-A., Olsson, G., Torbert, R. B., King, B., Granoff, M., Rau, D., et al. (2016). The spin-plane double probe electric field instrument for MMS. Space Science Reviews, 199(1-4), 137-165. https://doi.org/10.1007/s11214-014-0116-9

Liu, C. M., Fu, H. S., Xu, Y., Khotyaintsev, Y. V., Burch, J. L., Ergun, R. E., et al. (2018). Electron-scale measurements of dipolarization front. Geophysical Research Letters, 45, 4628-4638. https://doi.org/10.1029/2018GL077928

Nakamura, M., \& Scholer, M. (2000). Structure of the magnetopause reconnection layer and of flux transfer events: Ion kinetic effects. Journal of Geophysical Research, 105, 23,179-23,191. https://doi.org/10.1029/2000JA900101 
Oka, M., Fujimoto, M., Shinohara, I., \& Phan, T. D. (2010). "Island surfing” mechanism of electron acceleration during magnetic reconnection. Journal of Geophysical Research, 115, A08223. https://doi.org/10.1029/2010JA015392

Oka, M., Phan, T. D., Krucker, S., Fujimoto, M., \& Shinohara, I. (2010). Electron acceleration by multi-island coalescence. The Astrophysical Journal, 714(1), 915-926. https://doi.org/10.1088/0004-637x/714/1/915

Omura, Y., Matsumoto, H., Miyake, T., \& Kojima, H. (1996). Electron beam instabilities as generation mechanism of electrostatic solitary waves in the magnetotail. Journal of Geophysical Research, 101, 2685-2697. https://doi.org/10.1029/95JA03145

Pollock, C., Moore, T., Jacques, A., Burch, J., Gliese, U., Saito, Y., et al. (2016). Fast plasma investigation for Magnetospheric Multiscale. Space Science Reviews, 199(1-4), 331-406. https://doi.org/10.1007/s11214-016-0245-4

Pritchett, P. L. (2008). Energetic electron acceleration during multi-island coalescence. Physics of Plasmas, 15(10), 102105. https://doi.org/ $10.1063 / 1.2996321$

Retinò, A., Nakamura, R., Vaivads, A., Khotyaintsev, Y., Hayakawa, T., Tanaka, K., et al. (2008). Cluster observations of energetic electrons and electromagnetic fields within a reconnecting thin current sheet in the Earth's magnetotail. Journal of Geophysical Research, 113, A12215. https://doi.org/10.1029/2008JA013511

Russell, C. T., Anderson, B. J., Baumjohann, W., Bromund, K. R., Dearborn, D., Fischer, D., et al. (2016). The Magnetospheric Multiscale magnetometers. Space Science Reviews, 199(1-4), 189-256. https://doi.org/10.1007/s11214-014-0057-3

Slavin, J. A., Lepping, R. P., Gjerloev, J., Fairfield, D. H., Hesse, M., Owen, C. J., et al. (2003). Geotail observations of magnetic flux ropes in the plasma sheet. Journal of Geophysical Research, 108(A1), 1015. https://doi.org/10.1029/2002JA009557

Sonnerup, B. U. O., \& Scheible, M. (1998). Minimum and maximum variance analysis. In G. Paschmann \& P. W. Daly (Eds.), Analysis Methods for Multi-Spacecraft Data, No. SR-001 in ISSI Scientific Reports (Chap. 1, pp. 185-220). Noordwijk, Netherlands: ESA Publications Division.

Vaivads, A., Khotyaintsev, Y., André, M., \& Treumann, R. (2006). Plasma waves near reconnection sites. Lecture Notes in Physics, 687, 251-269. https://doi.org/10.1007/3-540-33203-0_10

Wang, R., Lu, Q., Li, X., Huang, C., \& Wang, S. (2010). Observations of energetic electrons up to $200 \mathrm{keV}$ associated with a secondary island near the center of an ion diffusion region: A cluster case study. Journal of Geophysical Research, 115, A11201. https://doi.org/10.1029/ 2010JA015473

Wang, R., Lu, Q., Nakamura, R., Huang, C., Li, X., Wu, M., et al. (2016). Electrostatic and electromagnetic fluctuations detected inside magnetic flux ropes during magnetic reconnection. Journal of Geophysical Research: Space Physics, 121, 9473-9482. https://doi.org/ 10.1002/2016JA022906

Wang, R. S., Lu, Q., Nakamura, R., Huang, C., Du, A., Guo, F., et al. (2016). Coalescence of magnetic flux ropes in the ion diffusion region of magnetic reconnection. Nature Physics, 12(3), 263-267. https://doi.org/10.1038/nphys3578

Zhao, Y., Wang, R., \& Du, A. (2016). Characteristics of field-aligned currents associated with magnetic flux ropes in the magnetotail: A statistical study. Journal of Geophysical Research: Space Physics, 121, 3264-3277. https://doi.org/10.1002/2015JA022144

Zhao, Y., Wang, R., Lu, Q., Du, A., Yao, Z., \& Wu, M. (2016). Coalescence of magnetic flux ropes observed in the tailward high-speed flows. Journal of Geophysical Research: Space Physics, 121, 10,898-10,909. https://doi.org/10.1002/2016JA023526

Zhong, J., Pu, Z. Y., Dunlop, M. W., Bogdanova, Y. V., Wang, X. G., Xiao, C. J., et al. (2013). Three-dimensional magnetic flux rope structure formed by multiple sequential X-line reconnection at the magnetopause. Journal of Geophysical Research: Space Physics, 118, 1904-1911. https://doi.org/10.1002/jgra.50281

Zhou, M., Berchem, J., Walker, R. J., el-Alaoui, M., Deng, X., Cazzola, E., et al. (2017). Coalescence of macroscopic flux ropes at the subsolar magnetopause: Magnetospheric Multiscale observations. Physical Review Letters, 119(5), 055101. https://doi.org/10.1103/ PhysRevLett.119.055101

Zhou, M., Deng, X. H., \& Huang, S. Y. (2012). Electric field structure inside the secondary island in the reconnection diffusion region. Physics of Plasmas, 19(4), 042902, https://doi.org/10.1063/1.3700194

Zhou, M., Pang, Y., Deng, X., Huang, S., \& Lai, X. (2014). Plasma physics of magnetic island coalescence during magnetic reconnection. Journal of Geophysical Research: Space Physics, 119, 6177-6189. https://doi.org/10.1002/2013JA019483

Zong, Q. G., Fritz, T. A., Pu, Z. Y., Fu, S. Y., Baker, D. N., Zhang, H., et al. (2004). Cluster observations of earthward flowing plasmoid in the tail. Geophysical Research Letters, 31, L18803. https://doi.org/10.1029/2004GL020692 